\title{
Value of quantitative analysis of left ventricular systolic function in patients on maintenance hemodialysis based on myocardial work technique
}

\author{
Chang Liu, Yi-Ping Feng ${ }^{*}$ (D, Zi-Ning Yan, Li Fan, Yi-Fei Rui and Ling Cui
}

\begin{abstract}
Background: This study aimed to determine the left ventricular (LV) systolic function in patients on maintenance hemodialysis (MHD) using the myocardial work (MW) technique and investigate the clinical value of the MW technique for the quantitative analysis of left ventricular (LV) systolic function in MHD patients with left ventricular hypertrophy $(\mathrm{LVH})$.

Methods: A total of $68 \mathrm{MHD}$ patients and 35 controls were registered in this study. The MHD patients were divided into the non-left ventricular hypertrophy $(\mathrm{NLVH})$ group $(n=35)$ and the LVH group $(n=33)$ according to the LV mass index (LVMI). MW was used to generate the LV global longitudinal strain (GLS), global work index (GWI), global constructive work (GCW), and global wasted work (GWW), global work efficiency (GWE). GLS and the MW parameters (GWI, GCW, GWW, GWE) were compared between groups and the correlations between these parameters and the LV ejection fraction (LVEF) in the LVH group were examined. The receiver operating characteristic (ROC) curve was used to evaluate the efficacy of MW parameters and GLS for the assessment of LV systolic dysfunction in MHD with LVH patients.

Results: The LVH group had significantly lower GWE, GWI, GCW, and GLS but higher GWW than the control and NLVH groups. Compared with the control group, the NLVH group had significantly lower GWE and GLS and higher GWW, but no significant differences in GWI, GCW were observed between these two groups. The LVEF was negatively correlated with GWW in MHD patients, but positively correlated with GWI, GWE, and GCW in the LVH group. Receiver operating characteristic curve (ROC) analysis revealed that GWE, GWW, GWI, and GCW had appreciable area under the curve (AUC), sensitivity, and specificity for evaluating LV function in LVH patients on MHD.
\end{abstract}

Conclusions: The MW parameters can quantitatively represent the LV myocardial work in MHD patients. Thus, the technique provides a new method for the quantitative evaluation of LV systolic function in MHD with LVH patients.

Keywords: Echocardiography, Maintenance hemodialysis, Myocardial work, Left ventricular hypertrophy, Systolic function

\section{*Correspondence: cccc5288@qq.com}

Department of Echocardiography, The Affiliated Changzhou No. 2

People's Hospital of Nanjing Medical University, Changzhou 213003, China

\section{Background}

Left ventricular (LV) systolic and diastolic dysfunction, LV hypertrophy (LVH) and myocardial fibrosis are the main features of cardiomyopathy in patients with endstage renal disease (ESRD) [1,2]. Maintenance hemodialysis (MHD) is the mainstay of treatment for ESRD 
patients. However, Lagies et al. [3] suggested that the degree of LVH and LV systolic dysfunction continue to worsen despite the prolongation of hemodialysis time and that MHD is unable to completely improve LV myocardial damage. $\mathrm{LVH}$ is the main cardiovascular disease in MHD patients due to changes in the workload of the heart $[4,5]$ and is a strong predictor of cardiovascular morbidity and mortality in this population [6]. Thus, early detection and diagnosis of LV dysfunction as well as $\mathrm{LVH}$ in MHD patients are of great significance for the administration of proper treatments, thus reducing mortality.

Two-dimensional speckle tracking imaging (2D-STI) technology is an effective tool for evaluating LV systolic function [7]; it can semi-automatically analyze LV myocardial strain, which sensitively reflects changes in LV function and is an appreciable prognostic indicator of cardiac adverse events. However, 2D-STI is load-dependent, i.e. it cannot explain how load changes affect the LV myocardial strain [8]. For example, increased afterload may cause lower LV strain, leading to misjudgment of the real LV systolic function [9]. Myocardial work (MW), which is derived from 2D-STI, combines the LV pressure (derived from the systolic pressure in the brachial artery) measured using a noninvasive method and the longitudinal strain data for the LV myocardium to establish the LV pressure-strain loops (PSL) [10]. This approach can be used to examine the changes in LV MW when LV becomes deformed due to overcoming the afterload.

Currently, MW is used in the evaluation of the effects of cardiac resynchronization therapy on hypertension, primary cardiomyopathy, and coronary heart disease (CHD) $[11,12]$. There are few reports on the use of MW in the evaluation of LV function in MHD patients. This study aimed to evaluate LV MW in patients with MHD. Our findings provide direct evidence of a new evaluation method for the early diagnosis of myocardial dysfunction in MHD patients.

\section{Methods}

\section{Ethics statement}

This study was approved by the Ethics Committee of Changzhou Second People's Hospital affiliated with Nanjing Medical University(Changzhou, China). The informed consent form was signed by all participants.

\section{Participant selection}

A total of 68 MHD patients with ESRD who were treated at the Second People's Hospital of Changzhou between January 2019 and November 2020 were retrospectively enrolled in this study. The inclusion criteria were as follows: (1) the primary disease was kidney disease and was diagnosed as ESRD; (2) all patients had a forearm arteriovenous anastomosis fistula and had dialysis thrice a week for 4 hours each time, which lasted for 6-36 months; and (3) patients did not have the following diseases: heart valve disease, CHD, congenital heart disease, primary cardiomyopathy, arrhythmia, and pericardial effusion. According to the LV mass index (LVMI) [13], the 68 patients were divided into two groups: the nonleft ventricular hypertrophy group $(\mathrm{NLVH})(\mathrm{n}=35)$, and the LVH group $(n=33)$. Thirty-five healthy subjects with matching age and sex who had no history of heart disease, hypertension, abnormal liver and kidney function, and diabetes were enrolled in the control group. All controls had normal physical indexes, electrocardiograms, and echocardiograms.

\section{Data collection}

The demographic and baseline clinical data for all participants were obtained from interviews and the hospital database.

\section{Conventional echocardiography}

All participants underwent conventional two-dimensional Doppler echocardiography (Vivid E9, GE Healthcare, Horten, Norway). MHD patients were examined within 30 minutes after hemodialysis and brachial artery blood pressure was recorded. The following echocardiographic parameters were also recorded: LV internal diameter at end diastole (LVIDD), LV internal diameter at end systole (LVIDS), interventricular septal diameter (IVSD), LV posterior wall diameter (LVPWD), and left atrium diameter (LAD). Left ventricular mass $(\mathrm{LVM})$ was calculated as follows: $\mathrm{LVM}=0.8 \times 1.04 \times$ $\left[\left(\right.\right.$ LVIDD + LVPWD + IVSD) ${ }^{3}-$ LVIDD $\left.^{3}\right]+0.6$, body surface area $(\mathrm{BSA})=0.0061 \times$ height $+0.0128 \times$ weight -0.1529 . LVM index $(\mathrm{LVMI})=\mathrm{LVM} / \mathrm{BSA}$. Blood flow velocity ( $E$ and $A)$ in the early and late diastolic mitral orifice was determined using pulsed Doppler and the E/A ratio was calculated. The peak speed (e') at the early mitral annulus motion was determined and the $\mathrm{E} / \mathrm{e}^{\prime}$ ratio was calculated. Images were acquired for offline analysis: the subject was connected to the electrocardiogram with an image frame rate of 60-90 frames per second. The blood flow spectrum for the mitral valve and aortic valve ostium was collected, and the two-, three-, and four-chamber views during three consecutive cycles were stored on the hard disk.

\section{Automated function imaging (AFI)}

The dynamic images of LV obtained from two-, three-, and four-chamber views as well as the images of blood stream spectrum from the mitral and aortic valves were imported into GE's offline cardiac analysis software (EchoPac PC version 203, GE Healthcare, Horten, 
Norway). After determining the opening and closing times of the mitral and aortic valves on the spectrum images, AFI was used to sequentially delineate the LV endocardial system in the three-, four-, and two-chamber views, and automatically depicted the LV myocardium as a region of interest (ROI). We then manually adjusted the envelope curve to match the wall of the chamber. The system automatically provided the longitudinal strain curve for the entire LV including $17 \mathrm{LV}$ segments, as well as the LV global longitudinal strain (GLS) and peak strain dispersion (PSD). The following two-plane LV volume parameters were obtained by pressing the ejection fraction (EF) button: LV end diastolic volume (LVEDV), LV end systolic volume (LVESV), and LVEF. The formula for LVEF was (LVEDV - LVESV)/ LVEDV $\times 100 \%$.

GLS and several MW parameters, namely global work index (GWI), global constructive work (GCW), global wasted work (GWW), and global work efficiency (GWE), were obtained through the following sequential steps: (1) clicking the "myocardial work" button, (2) input of brachial artery blood pressure value, and (3) clicking the "Advanced" button. The system constructed an LV PSL based on the isovolumic contraction, ejection phase, and isovolumic relaxation phases, defined by the valve opening and closing times.

\section{Evaluation of intra- and inter-observer variability}

We randomly selected 30 subjects from the control group, the NLVH group, and the LVH group and compared the differences between two observations made by the same observer after one week. The differences between two independent observers who were unaware of the patient groupings were also evaluated. Interclass correlation coefficients (ICCs) were used to evaluate the intra- and inter-observer variability. The criteria for ICCs were: "excellent" if $\geq 0.80$, "good" if $0.61-0.79$, "moderate" if $0.41-0.60$, and "poor" if $\leq 0.40$.

\section{Statistical analysis}

SPSS 22.0 (IBM SPSS, Statistics, Chicago, IL, USA) statistical software was used for all statistical analyses. The measurement data are expressed as mean \pm standard deviation (SD), and the count data are expressed as $\mathrm{n}(\%)$. Data normality was tested using Kolmogorov-Smirnov's method. The single-factor analysis of variance was used for the comparison of normally distributed data among three groups, while the Student-Newman-Keuls (SNK) method was used to compare data between two groups. The Kruskal-Wallis method was used to compare nonnormally distributed data. The rates in the general data were compared using the chi-square test or corrected chi-square test. $P<0.05$ was considered statistically significant. For determination of the correlation between
LVEF and the MW parameters including GWI, GWE, GCW, and GWW in MHD with LVH patients, Pearson correlation analysis was used for normally distributed variables, and Spearman correlation analysis was used for non-normally distributed variables. The receiver operating characteristic curve (ROC) was used to analyze the diagnostic efficacy of GWE, GWI, GCW, GWW, and GLS for LV systolic function in MHD with LVH patients. The Youden index was used to calculate the most appropriate cutoff for each value and to obtain the specificity and sensitivity. The criteria for the areas under the curves (AUCs) were: "good" if 0.90-1.00, "moderate" if 0.710.89 , "poor" if $0.51-0.70$, and "useless" if $\leq 0.50$.

\section{Results}

Demographic and baseline clinical characteristics of participants

We first compared the demographic and baseline clinical characteristics of participants in the control group $(\mathrm{n}=35$; men: 19 ; women: 16$)$, the NLVH group $(\mathrm{n}=35$; men: $18, L V M I \leq 115 \mathrm{~g} / \mathrm{m}^{2}$; women: $17, \mathrm{LVMI} \leq 95 \mathrm{~g} /$ $\left.\mathrm{m}^{2}\right)$, and the LVH group $(\mathrm{n}=33$; men: 19, LVMI $>115 \mathrm{~g} /$ $\mathrm{m}^{2}$; women: 14 , LVMI $>95 \mathrm{~g} / \mathrm{m}^{2}$ ). As shown in Table 1 , no significant differences were observed with regard to age, sex, heart rate, and body mass index (BMI) between the groups (all $P>0.05$ ). Further, there were no significant differences in remove volume, dry weight, cause of disease, and medication history between these three groups. As expected, the NLVH and LVH groups had significantly higher systolic blood pressure (SBP), diastolic blood pressure (DBP), and creatinine levels than the control group $(P<0.05)$, but no significant differences in these parameters were observed between the NLVH and LVH groups $(P>0.05)$. The $\mathrm{N}$-terminal pro-brain natriuretic peptide (NT-proBNP) values in the NLVH group and LVH group were significantly higher than those in the control group, and the NT-proBNP levels in the LVH group were significantly higher than those in the NLVH group. In addition, the $\mathrm{LVH}$ group had a significantly longer duration of dialysis than the NLVH group $(P<0.05)$.

\section{Conventional echocardiographic parameters for participants}

We next compared the conventional echocardiographic parameters between these three groups. Compared with the control group, the NLVH and LVH groups had significantly higher LVIDD, LVIDS, LAD, LVEDV, and LVESV $(P<0.05)$, but there were no significant differences in these parameters between the NLVH and LVH groups $(P>0.05)$. In addition, the $\mathrm{LVH}$ group had a significantly lower LVEF than the control group $(\mathrm{P}<0.05)$. The LVH group had significantly worse E/A and E/e' than the control and NLVH groups, while the NLVH group had 
Table 1 Demographic and baseline clinical characteristics of participants

\begin{tabular}{|c|c|c|c|c|}
\hline & Controls $(n=35)$ & NLNH $(n=35)$ & LVH $(n=33)$ & $P$ value \\
\hline Age (years) & $51.34 \pm 8.10$ & $52.11 \pm 7.95$ & $51.94 \pm 7.50$ & 0.913 \\
\hline Male sex (number) & $19(54.3 \%)$ & $18(51.4 \%)$ & $18(54.5 \%)$ & 0.959 \\
\hline Heart rate (beats/min) & $71.77 \pm 9.86$ & $75.66 \pm 7.92$ & $75.94 \pm 9.23$ & 0.069 \\
\hline $\mathrm{BMI}\left(\mathrm{kg} / \mathrm{m}^{2}\right)$ & $22.51 \pm 2.20$ & $22.17 \pm 1.95$ & $22.09 \pm 1.76$ & 0.651 \\
\hline HD time (months) & & $12 \pm 3$ & $27 \pm 5^{\#}$ & $<0.001$ \\
\hline Remove volume (kg) & & $1.88 \pm 1.00$ & $52.55 \pm 8.34$ & 0.143 \\
\hline Dry weight (kg) & & $50.80 \pm 7.93$ & $2.21 \pm 0.80$ & 0.380 \\
\hline $\mathrm{SBP}(\mathrm{mmHg})$ & $120.06 \pm 5.72$ & $146.06 \pm 23.98^{*}$ & $148.18 \pm 15.86^{*}$ & $<0.001$ \\
\hline $\mathrm{DBP}(\mathrm{mmHg})$ & $69.43 \pm 5.94$ & $86.40 \pm 10.63^{*}$ & $89.64 \pm 9.16^{*}$ & $<0.001$ \\
\hline Creatinine ( $\mu \mathrm{mol} / \mathrm{L})$ & $63.86 \pm 9.44$ & $821.89 \pm 182.04^{*}$ & $877.15 \pm 177.15^{*}$ & $<0.001$ \\
\hline NT-proBNP (pg/ml) & $66.74 \pm 22.18$ & $1212.06 \pm 419.68^{*}$ & $14551.06 \pm 10831.02^{* \#}$ & $<0.001$ \\
\hline NYHA II-IV (cases) & & $8(23 \%)$ & $32(97 \%)^{\#}$ & $<0.001$ \\
\hline \multicolumn{5}{|l|}{ Cause of ESRD } \\
\hline Glomerulonephritis (cases) & & $17(48.6 \%)$ & $17(51.5 \%)$ & 0.808 \\
\hline Diabetic Nephropathy (cases) & & $9(25.7 \%)$ & $7(21.2 \%)$ & 0.662 \\
\hline Hypertensive nephrosclerosis (cases) & & $4(11.4 \%)$ & $4(12.1 \%)$ & 0.929 \\
\hline Polycystic kidney (cases) & & $3(8.6 \%)$ & $2(6.1 \%)$ & 0.692 \\
\hline Unknown (case) & & $2(5.7 \%)$ & $3(9.1 \%)$ & 0.594 \\
\hline \multicolumn{5}{|l|}{ Medication } \\
\hline ACEI/ARB (case) & & $18(51 \%)$ & $21(64 \%)$ & 0.309 \\
\hline Calcium channel blockers (case) & & $22(63 \%)$ & $24(73 \%)$ & 0.385 \\
\hline$\beta$-Receptor blockers (case) & & $14(40 \%)$ & $16(49 \%)$ & 0.481 \\
\hline
\end{tabular}

Data are expressed as mean \pm SD or number of subjects (\%). Bold number: $P<0.05 .{ }^{*} P<0.05$, vs. normal. ${ }^{~} P<0.05$, vs. NLVH. BMI, body mass index; HD, hemodialysis; SBP, systolic blood pressure; DBP, diastolic blood pressure; NT-proBNP, N-terminal pro-brain natriuretic peptide; ESRD: end-stage renal disease

significantly worse E/A and E/e' than the control group, indicating that MHD patients, especially those in the LVH group, had diastolic dysfunction (Table 2).

\section{Comparison of MW and strain parameters of participants}

The NLVH and LVH groups had lower GWE and GLS but higher GWW than the control group $(\mathrm{P}<0.05)$. The LVH group had lower GWE and GLS but higher GWW and PSD than the NLVH group $(\mathrm{P}<0.05)$. In addition, the LVH group had significantly lower GWI and GCW than both the control and NLVH groups $(\mathrm{P}<0.01)$, and had higher PSD than the control group. However, the control and NLVH groups had comparable GWI, GCW, and PSD $(\mathrm{P}>0.05)$ (Table 3).

The control group had the largest PSL area and the smallest global average wasted work. The color of the bull's eye in the control group was uniformly green, suggesting high work efficiency. The PSL area and global average wasted work for the NLVH group were between those for the control and LVH groups. The bull's eye was unevenly light yellow, indicating a slightly lower work efficiency. The color of the bull's eye in the LVH group was messy, with yellow and orange colors, indicating low work efficiency (Fig. 1).
Table 2 Conventional for participants

\begin{tabular}{|c|c|c|c|c|}
\hline & $\begin{array}{l}\text { Controls } \\
(n=35)\end{array}$ & $\begin{array}{l}\text { NLVH } \\
(n=35)\end{array}$ & $\begin{array}{l}\text { LVH } \\
(n=33)\end{array}$ & $P$ value \\
\hline LVIDD (mm) & $46.17 \pm 3.14$ & $50.80 \pm 5.30^{*}$ & & \\
\hline LVIDS (mm) & $30.86 \pm 2.76$ & $35.43 \pm 4.45^{*}$ & $37.15 \pm 4.58^{*}$ & $<0.001$ \\
\hline IVSD (mm) & $8.77 \pm 1.42$ & $9.06 \pm 1.35$ & & $<0.001$ \\
\hline LVPWD (mm) & $8.94 \pm 1.53$ & $9.23 \pm 1.59$ & $11.76 \pm 1.30^{* \#}$ & $<0.001$ \\
\hline $\operatorname{LAD}(\mathrm{mm})$ & $34.80 \pm 2.74$ & $40.69 \pm 5.15^{*}$ & $42.76 \pm 4.97^{*}$ & $<0.001$ \\
\hline LVEDV (ml) & $69.26 \pm 10.37$ & $94.77 \pm 32.49^{*}$ & $106.85 \pm 30.89^{*}$ & $<0.001$ \\
\hline LVESV (ml) & $28.89 \pm 5.70$ & $44.31 \pm 19.40^{*}$ & $57.03 \pm 23.24^{*}$ & $<0.001$ \\
\hline LVEF (\%) & $58.23 \pm 3.37$ & $55.63 \pm 8.92$ & $52.67 \pm 8.19^{*}$ & $<0.001$ \\
\hline$E / A$ & $1.15 \pm 0.19$ & $0.99 \pm 0.15^{*}$ & $0.62 \pm 0.18^{* \#}$ & $<0.001$ \\
\hline$E / e^{\prime}$ & $7.08 \pm 1.36$ & $8.86 \pm 1.36^{*}$ & $12.22 \pm 3.60^{* \#}$ & $<0.001$ \\
\hline
\end{tabular}

Data are expressed as mean \pm SD. Bold number: $P<0.05 .{ }^{*} P<0.05$, vs normal. ${ }^{\#} P<0.05$, vs NLVH. LVIDD left ventricular internal diameter at end-diastole; LVIDS left ventricular internal diameter at end-systole; IVSD interventricular septal diameter; LVPWD left ventricular posterior wall diameter; LAD left atrial diameter; LVEDV left ventricular end-diastolic volume; LVESV left ventricular end-systolic volume; LVEF left ventricular ejection fraction; E/A: peak early diastolic velocity of mitral orifice/peak late diastolic velocity of mitral orifice (by pulsed Doppler); E/e': peak early diastolic velocity of mitral orifice (by pulsed Doppler)/peak velocity during early diastolic of mitral annulus (by pulsed-wave tissue Doppler) 
Table 3 MW and strain parameters for participants

\begin{tabular}{|c|c|c|c|c|}
\hline & $\begin{array}{l}\text { Controls } \\
(n=35)\end{array}$ & $\begin{array}{l}\text { NLVH } \\
(n=35)\end{array}$ & $\begin{array}{l}\text { LVH } \\
(n=33)\end{array}$ & $P$ value \\
\hline GWE (\%) & $96.20 \pm 1.43$ & $90.66 \pm 4.19^{*}$ & $86.42 \pm 4.63^{* \#}$ & $<0.001$ \\
\hline GWI (mmHg\%) & $2137.46 \pm 220.80$ & $2171.34 \pm 321.37$ & $1781.18 \pm 282.39^{* \#}$ & $<0.001$ \\
\hline GCW (mmHg\%) & $2236.34 \pm 238.44$ & $2308.14 \pm 339.81$ & $1954.79 \pm 275.42^{* \#}$ & $<0.001$ \\
\hline GWW (mmHg\%) & $76.69 \pm 30.60$ & $199.26 \pm 94.31^{*}$ & $256.64 \pm 92.72^{* \#}$ & $<0.001$ \\
\hline GLS (\%) & $-20.66 \pm 1.78$ & $-16.87 \pm 2.70^{*}$ & $-14.67 \pm 2.94^{* \#}$ & $<0.001$ \\
\hline PSD (ms) & $42.06 \pm 9.43$ & $59.97 \pm 21.39$ & $79.88 \pm 18.45^{* \#}$ & $<0.001$ \\
\hline
\end{tabular}

Data are expressed as mean \pm SD. Bold number: $P<0.05 .{ }^{*} P<0.05$, vs. Normal. ${ }^{\#} P<0.05$, vs. NLVH. GWE: global work efficiency; GWl: global work index; GCW: global constructive work; GWW: global wasted work; GLS: global longitudinal strain; PSD: peak strain dispersion

\section{Definition of abnormal left ventricular function in LVH group}

According to the diagnostic criteria for heart failure with preserved ejection fraction (HFpEF) proposed by the European Society of Cardiology in 2019 [14], the LVEF in the LVH group was normal or slightly reduced. However, these patients had cardiac function classes of II-IV according to the New York Heart Association (NYHA) cardiac function classification, and NT-proBNP>125 pg/ $\mathrm{ml}, \mathrm{GLS}<16 \%$, LVH and/or left atrium enlargement, and diastolic dysfunction. Therefore, the patients in the LVH group had abnormal LV function.

\section{Correlation between MW parameters and LVEF in MHD with LVH patients}

We performed correlation analyses between the MW parameters and LVEF in the LVH group; the latter is a widely used indicator of cardiac function [15]. We found that LVEF was positively correlated with GWI, GWE, and GCW $(r=0.643,0.523,0.505$; all $\mathrm{P}<0.05)$, but negatively correlated with GWW ( $\mathrm{r}=-0.506, \mathrm{P}=0.003)$ (Fig. 2).

\section{Determination of the cutoff values, sensitivity, and specificity of MW parameters and GLS for identifying LV function in MHD with LVH patients}

We next used AUC curves to determine the sensitivity and specificity of MW parameters and GLS for identifying LV function in MHD with $\mathrm{LVH}$ patients. GWE, GWW, and GLS had AUCs of 0.982, 0.972, and 0.971, respectively (Fig. 3a, b, c), with respective cutoff values of 94\%, $123 \mathrm{mmHg} \%$, and - 18.44\%. GWE had higher sensitivity than GLS and GWW (97.0\% vs. $93.0 \%$ and $84.9 \%)$. In addition, GWW has higher specificity than GLS and GWE (97.1\% vs. 94.3\% and 91.4\%). The AUCs for GWI (Fig. 3d) and GCW (Fig. 3e) were 0.835 and 0.782, respectively, both of which were lower than the AUCs for GWE, GWW, and GLS.
Intra- and inter-observer variability

We also examined the intra- and inter-observer variability in this study. The intra-observer differences in the ICC values of GLS, PSD, LVEF, GWI, GCW, GWW, and GWE were $0.94,0.93,0.84,0.86,0.88,0.96$, and 0.88 , respectively. The differences in the ICC values for these parameters between the observers were 0.93, 0.82, 0.79, $0.91,0.89,0.93$, and 0.82 , respectively. The ICC value and $95 \%$ confidence interval for each parameter are shown in Table 4. Our results suggested that our study generated reliable and consistent observations.

\section{Discussion}

In the present study, we examined the feasibility of MW parameters for evaluating the LV function in MHD patients with LVH and found that GWI, GCW, GWW, and GWE had appreciable AUC, specificity, and sensitivity in the LVH group. Therefore, we believe that these MW parameters may be reliably used to accurately evaluate the LV function in MHD patients, especially in those with LVH.

In this study, we first analyzed the conventional echocardiographic parameters for participants in the three groups and found that the MHD patients underwent LV remodeling, a structural basis for LV dysfunction [16, 17]. A previous study showed that there was no significant difference in LV diameter and wall thickness between non-hemodialysis and hemodialysis patients [18], indicating that hemodialysis does not significantly improve LV remodeling and cardiac contractile function in patients. On the contrary, with the prolongation of dialysis time and the aggravation of LV remodeling, the LV systolic function in MHD patients will be further impaired. The main feature of LV lesions in MHD patients is the progression of the compensatory phase of $\mathrm{LVH}$ to the decompensatory phase, because these patients have long-term pressure overload, including 


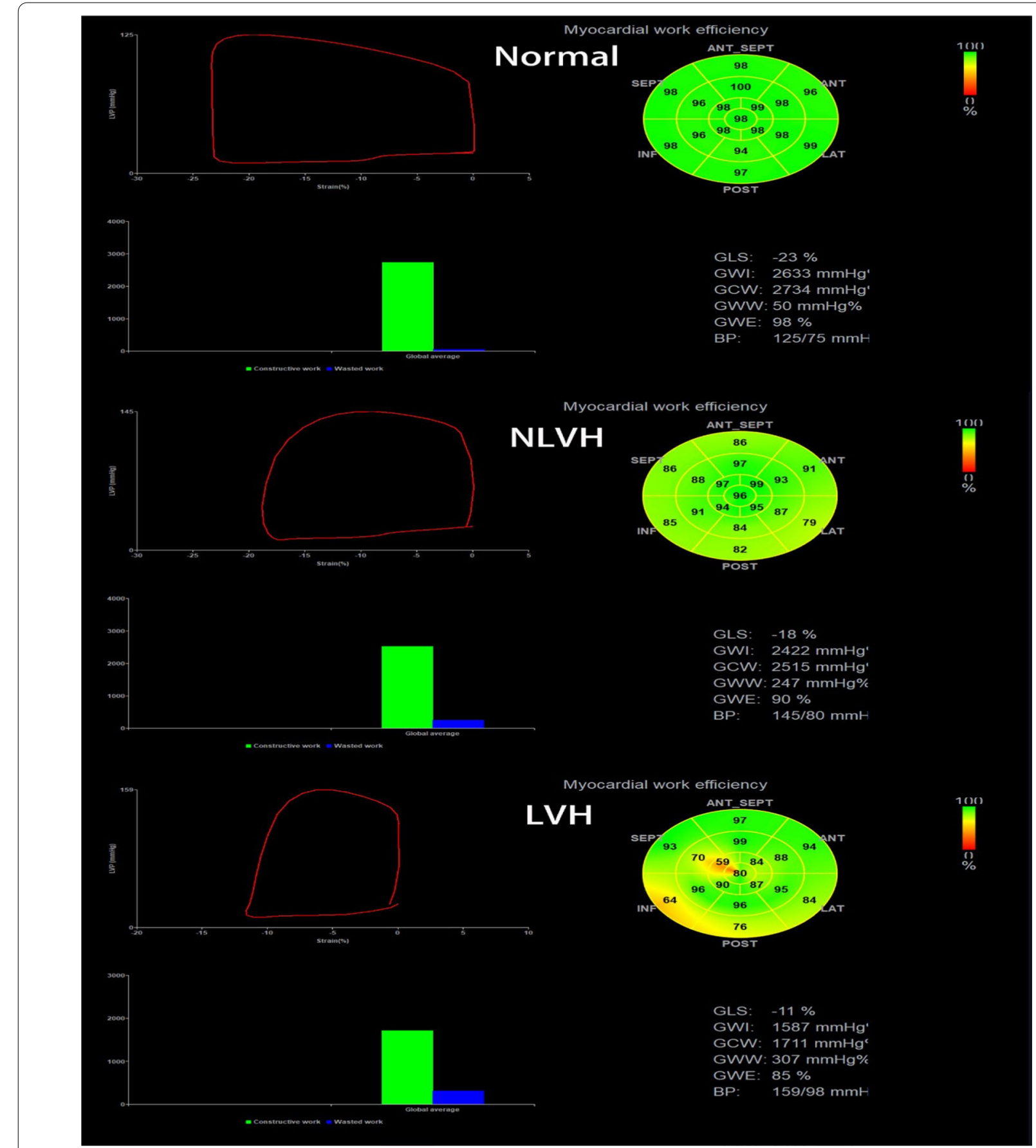

Myocardial work efficiency

Fig. 1 Comparison of left ventricular PSL and myocardial work parameters in control, NLVH, and LVH groups. The control group had the largest PSL area and good myocardial work parameters (GWE: 98\%, GWW: 50 mmHg\%, GWl: 2633 mmHg\%, GCW: 2734 mmHg\%). The NLVH group had a PSL area between those of the control and LVH groups, and some myocardial work parameters were poor (GWE: 90\%, GWW: 247 mmHg\%), while some were better (GWI: $2422 \mathrm{mmHg} \%, \mathrm{GCW}: 2515 \mathrm{mmHg} \%$ ). The LVH group had the smallest PSL area and worst myocardial work parameters (GWE: 85\%, GWW: 307 mmHg \%, GWl: 1587 mmHg\%, GCW: 1711 mmHg\%) 

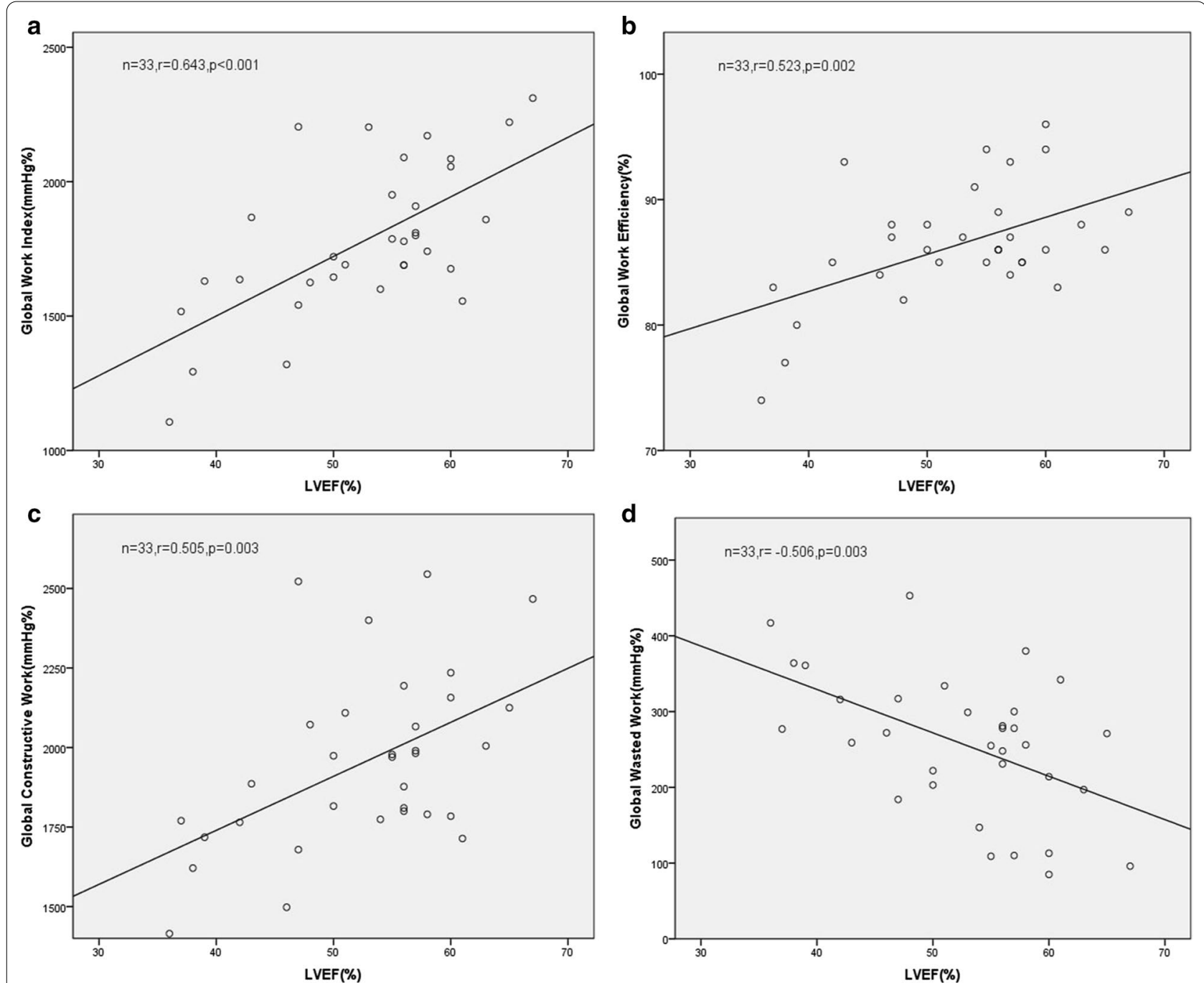

Fig. 2 Analysis of correlation of the MW parameters GWI (a), GWE (b), GCW (c), and GWW (d) with LVEF in MHD with LVH patients. The correlation coefficients were $0.643,0.523,0.505$, and -0.506 , respectively

chronic hypertension, anemia, secondary hyperparathyroidism, and arteriovenous fistula [19, 20].

Our further analysis revealed that $\mathrm{LVH}$ and NLVH patients had significantly lower GLS than the controls. Moreover, with the increase in the LV thickness and prolongation of the dialysis time, the GLS decreased significantly, which was accompanied by a decrease in the LV systolic function, as evidenced by the lower GLS in MHD with LVH patients than in NLVH patients. These findings were in line with our previous report [21]. GLS is usually obtained from 2D-STI technology, which quantitatively measures the mechanical parameters for LV function [22, 23]. GLS is considered to be a better indicator than EF and is widely used in clinics to assess LV function [24]. However, some scholars recently suggested that strain measurements are susceptible to load, which in turn affects the accurate assessment of myocardial function [25]. Therefore, it is important to use effective screening methods to verify the GLS.

Recently, the MW parameters derived from PSL have been used to determine LV function and shown to have a good agreement between the noninvasively measured PSL and the invasively directly measured PSL [26]. In this study, we examined the possibility of using MW parameters to assess LV function in MHD patients. We found that the LVH group had significantly lower GLS, GWI, GCW, and GWE but higher GWW than the control and NLVH groups. LVH patients have pathological cardiac changes including disordered arrangement of myocardial fibers and increased myocardial interstitial 

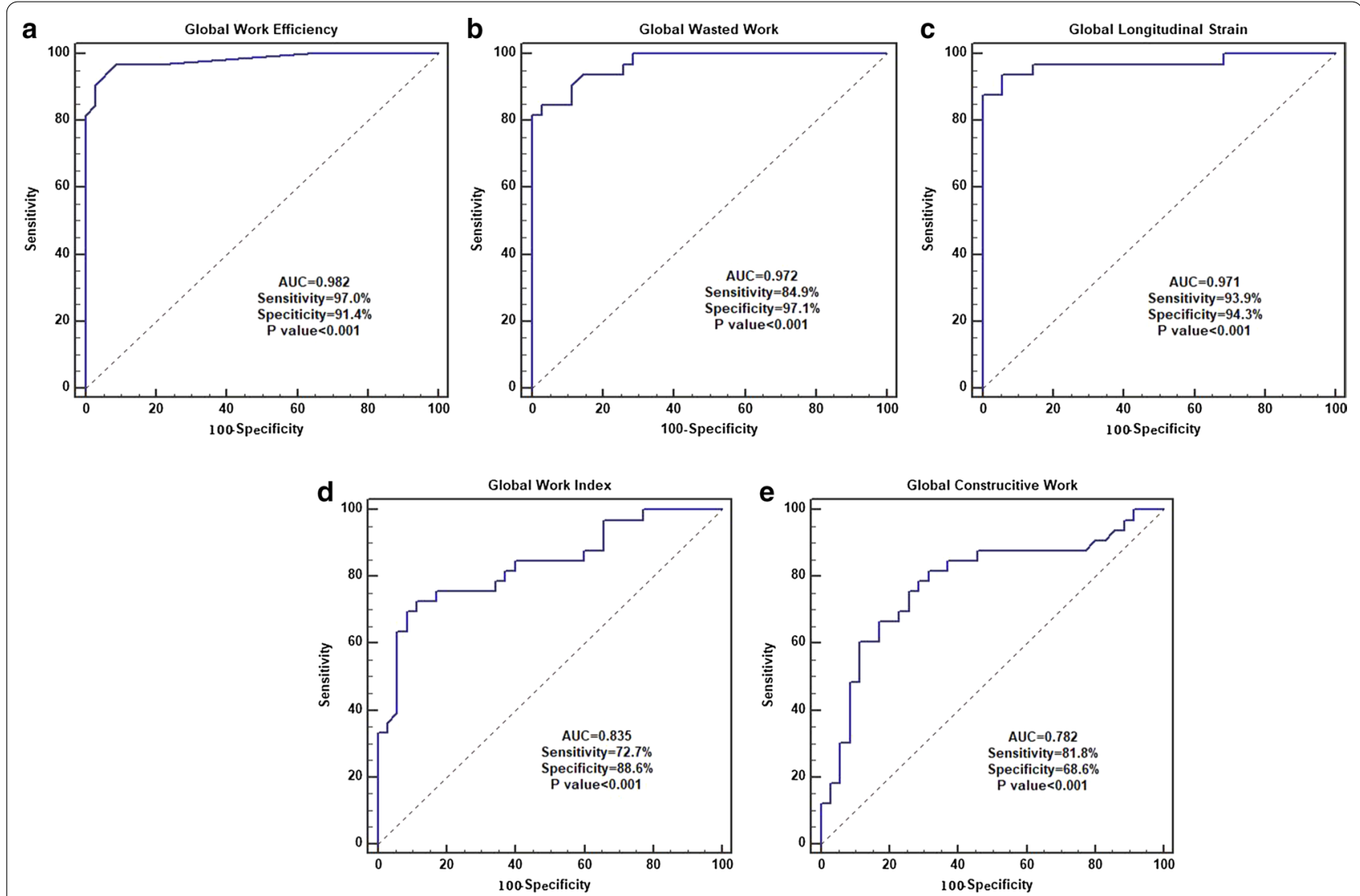

Fig. 3 ROC curve analysis to evaluate the sensitivity and specificity of GWE (a), GWW (b), GLS (c), GWI (d) and GCW (e) for identifying LV dysfunction in MHD with LVH patients. The area under the ROC curve values for the GWE, GWW, GLS, GWI, and GCW were $0.982,0.972,0.971,0.835$, and 0.782 , respectively. The sensitivity for the GWE, GWW, GLS, GWI, and GCW were $97.0 \%, 84.9 \%, 93.9 \%, 72.7 \%$, and $81.8 \%$, respectively. The specificity for the GWE, GWW, GLS, GWI, and GCW were $91.4 \%, 97.1 \%, 94.3 \%, 88.6 \%$, and $68.6 \%$, respectively. The cutoff values for the GWE, GWW, GLS, GWI, and GCW were $94 \%, 123 \mathrm{mmHg} \%,-18.44 \%, 1909 \mathrm{mmHg} \%$, and $2157 \mathrm{mmHg} \%$, respectively

Table 4 Intra- and inter-observer variability

\begin{tabular}{llllll}
\hline & \multicolumn{2}{l}{ Intra-observer } & & \multicolumn{2}{l}{ Inter-observer } \\
\cline { 2 - 3 } & ICC & $\mathbf{9 5 \%} \mathbf{C l}$ & & ICC & $\mathbf{9 5 \% ~ C l}$ \\
\hline GLS & 0.94 & $0.89-0.97$ & & 0.93 & $0.85-0.96$ \\
PSD & 0.93 & $0.86-0.97$ & & 0.82 & $0.66-0.91$ \\
LVEF & 0.84 & $0.69-0.92$ & & 0.79 & $0.61-0.89$ \\
GWI & 0.86 & $0.74-0.94$ & & 0.91 & $0.82-0.95$ \\
GCW & 0.88 & $0.76-0.94$ & & 0.89 & $0.79-0.95$ \\
GWW & 0.96 & $0.91-0.98$ & & 0.93 & $0.86-0.97$ \\
GWE & 0.88 & $0.77-0.94$ & & 0.82 & $0.65-0.91$ \\
\hline
\end{tabular}

ICC, intra-class correlation coefficients; $95 \% \mathrm{Cl}, 95 \%$ confidence interval; GLS, global longitudinal strain; PSD, peak strain dispersion; LVEF, left ventricular ejection fraction; GWI, global work index; GCW, global constructive work; GWW, global wasted work; GWE, global work efficiency

fibrosis due to damage to myocardial cells [27]. In addition, $\mathrm{LVH}$ reduces the density of myocardial microcirculation and myocardial blood supply [28], resulting in decreased GWI and GCW. Early studies have shown that GWI has a strong correlation with myocardial glucose metabolism, basically reflecting the local myocardial oxygen consumption [29,30]. Galli et al. found that patients with ischemic or dilated cardiomyopathy had increased GWI and GCW after cardiac resynchronization therapy $[31,32]$. Thus, GWI and GCW can be used as indicators of myocardial survival. It is noteworthy that GCW is also the main predictor of LV fibrosis [33].

In MHD with LVH patients, the LV myocardium is significantly damaged by the accumulated metabolites and increased myocardial fibrosis, resulting in the impairment of LV systolic function. In the normal heart, all myocardial segments contract in a synchronized manner, but MHD with LVH patients and those with myocardial fibrosis have altered electrophysiological characteristics in the myocardium, which causes myocardial excitation-contraction uncoupling [34]. Thus, the LV contraction is not synchronized in MHD with LVH patients, as reflected by the increased PSD. 
Hence, some LV segments elongate during contraction, significantly wasting MW, which is reflected by increased GWW. The decrease in GWE probably derived from increased GWW and decreased GCW in MHD with LVH patients, indicating that LVH in MHD patients can cause a significant reduction in LV systolic function.

The NLVH group had comparable GWI and GCW to the control group, but higher GWI and GCW than the LVH group. Since the degree of LV myocardial impairment in the NLVH patients was smaller than that in the LVH patients, LV remodeling and fibrosis were not as severe as those in the LVH patients. Thus, it is understandable that NLVH patients had higher GWI and GCW than the LVH patients. MHD patients usually have poorly controlled hypertension [35]. In order to counteract the increased afterload during contraction [11], the heart pump function is compensated by shifting to a higher energy level [36], which is evidenced by higher GWI and GCW. This also suggests that the LV myocardium was not significantly impaired in NLVH patients and that LVEF was maintained at a normal level by enhanced myocardial contractility. However, if the high GWI persists and exceeds the compensatory capacity of the myocardium, LV remodeling and dysfunction will occur [37]. In addition, the NLVH group had higher GWW but lower GWE than the control group, with no significant difference in the LV PSD between the groups. This suggests that the GWW of the LV begins to increase while the LV thickness and synchronicity are still within the normal range in MHD patients. However, due to a slight increase in GCW, GWE decreased slightly. In other words, MW increased in the NLVH patients to maintain the contractile function at a normal level, but myocardial ineffective work also increased, which can potentially lead to impaired myocardial systolic function.

LVEF is the most commonly used evaluator for LV systolic function in the clinic [38]. In the present study, we found that LVEF was positively correlated with GWI, GCW, and GWE, and negatively correlated with GWW in the LVH group. LV segments of the normal heart contract synchronously, and the MW generated by the myocardium to overcome the afterload can be quantified using GWI and GCW, both of which have a positive effect on LV ejection [32]. However, when GWW is at a low level, GWE should be close to 100\% [39]. As the thickness of the myocardium and the degree of myocardial injury increases in MHD patients, the decrease in LV systolic function is accompanied by an increase in GWW and a decrease in GWI, GCW, and GWE. MW can thus be used to quantitatively analyze the degree of LV injury in MHD with LVH patients, thus serving as a new sensitive indicator.
Our further analysis of the GLS and MW parameters for LVH patients revealed that the AUCs of GWE, GWW, and GLS were significantly higher than that of GWI and GCW. This observation was different from that in a previous study [40], in which the AUCs of GWI and GCW in CHD patients were higher than those of GLS, GWE, and GWW. This discrepancy may be explained by the different mechanisms underlying CHD and LVH in MHD patients. CHD is caused by the reduction or interruption of blood supply in the coronary arteries, leading to ischemia and necrosis of myocardial cells and early development of myocardial dysfunction [41]. This directly leads to a reduction in GWI and GCW. On the contrary, the damage from hemodialysis to the heart muscles is independent of the existence of coronary atherosclerosis [42]. Instead, the cardiac muscle damage in MHD patients is caused by the rapid changes in load [43], and the LV systolic function will only be significantly weakened during the decompensation period. Therefore, the test efficacy of GWI and GCW in MHD patients is relatively low. On the other hand, we found that the AUCs of GWE and GWW were higher than that of GLS. In addition, one of the main limitations of GLS is its loaddependence, and the increase in afterload can reduce the wall tension, leading to a misinterpretation of the true contractile function of the myocardium [40,44]. However, for MW measurement, the non-invasive PSL integrates the afterload into the LV strain parameters, and thus MW is an optimization of the strain values [45]. Hence, the conclusions obtained from using non-invasive PSL are more comprehensive and objective.

\section{Limitations}

The limitations of this study should be noted. First, this study only evaluated the global LV MW in MHD patients and the LV 17-segment MW parameters were not investigated. Second, MW technology mainly presents the effect of afterload on LV strain. The effect of pre-load on LV strain in MHD patients needs to be further investigated in the future. Third, this study was a single-center study with a small sample size. Thus, our conclusions should be corroborated in future studies performed in multiple centers with large cohorts.

\section{Conclusions}

We report here that the MW technique is an optimized 2D-STI technique and that the MW parameters, GWI, GCW, GWW, and GWE, can be used to assess LV function in MHD patients, especially in those with LVH.

\section{Abbreviations}

MW: Myocardial work; 2D-STI: Two-dimensional speckle tracking imaging; MHD: Maintenance hemodialysis; LVH: Left ventricular hypertrophy; ESRD: End 
stage renal disease; NT-proBNP: N-terminal pro-brain natriuretic peptide; LVMI: Left ventricular mass index; GLS: Global longitudinal strain; PSD: Peak strain dispersion; PSL: Pressure-strain loops; GWl: Global work index; GCW: Global constructive work; GWW: Global wasted work; GWE: Global work efficiency; LVEF: Left ventricular ejection fraction; LVIDD: Left ventricular internal diameter at end-diastole; LVIDS: Left ventricular internal diameter at end-systole; IVSD: Interventricular septal diameter; LVPWD: Left ventricular posterior wall diameter; LAD: Left atrial diameter; LVEDV: Left ventricular end-diastolic volume; LVESV: Left ventricular end-systolic volume.

\section{Acknowledgements}

Not applicable.

\section{Authors' contributions}

LC and FYP designed the study, collected and analyzed echocardiography measurements. LC wrote the manuscript. YZN and FYP revised the manuscript. $\mathrm{LC}$ and $\mathrm{CL}$ collected the clinical data. RYF and FL checked the validity of the data. $\mathrm{LC}$ and $\mathrm{FL}$ performed the statistical analysis. All authors have read and approved the final manuscript.

\section{Funding}

None.

\section{Availability of data and materials}

The datasets used and/or analyzed during the current study are available from the corresponding author on reasonable request.

\section{Ethics approval and consent to participate}

This study was approved by the Ethics Committee of Changzhou Second People's Hospital affiliated with Nanjing Medical University. The informed consent form was signed by all participants.

\section{Consent for publication}

Not applicable.

\section{Competing interests}

The authors declare that they have no competing interests.

Received: 2 July 2020 Accepted: 31 January 2021

Published online: 06 February 2021

\section{References}

1. Wang $X$, Shapiro Jl. Evolving concepts in the pathogenesis of uraemic cardiomyopathy. Nat Rev Nephrol. 2019;15:159-75.

2. Radhakrishnan A, Pickup L, Price A, Law J, Edwards N, Steeds R, Ferro C, Townend J. Coronary microvascular dysfunction: a key step in the development of uraemic cardiomyopathy? Heart. 2019;105(17):1302-9.

3. Lagies R, Beck B, Hoppe B, Sheta S, Weiß V, Sreeram N. Cate FUt: Inhomogeneous longitudinal cardiac rotation and impaired left ventricular longitudinal strain in children and young adults with end-stage renal failure undergoing hemodialysis. Echocardiography. 2015;32(8):1250-60.

4. Dörr K, Kammer M, Reindl-Schwaighofer R, Lorenz M, Loewe C, Marculescu R, Erben R, Oberbauer R. Effect of etelcalcetide on cardiac hypertrophy in hemodialysis patients: a randomized controlled trial (ETECAR-HD). Trials. 2019;20(1):601.

5. Foley RN, Parfrey PS. Risk factors for cardiac morbidity and mortality in dialysis patients. Curr Opin Nephrol Hypertens. 1994;3(6):608-14.

6. McCullough PA, Chan CT, Weinhandl ED, Burkart JM, Bakris GL. Intensive hemodialysis, left ventricular hypertrophy, and cardiovascular disease. Am J Kidney Dis. 2016;68(5s1):5-s14.

7. Collier P, Phelan D, Klein A. A Test in Context: Myocardial Strain Measured by Speckle-Tracking Echocardiography. J Am Coll Cardiol. 2017;69(8):1043-56.

8. Boe E, Russell K, Eek C, Eriksen M, Remme EW, Smiseth OA, Skulstad H. Non-invasive myocardial work index identifies acute coronary occlusion in patients with non-ST-segment elevation-acute coronary syndrome. Eur Heart J Cardiovasc Imaging. 2015;16(11):1247-55.
9. Chan J, Edwards NFA, Khandheria BK, Shiino K, Sabapathy S, Anderson B, Chamberlain R, Scalia GM. A new approach to assess myocardial work by non-invasive left ventricular pressure-strain relations in hypertension and dilated cardiomyopathy. Eur Heart J Cardiovasc Imaging. 2019;20(1):31-9.

10. Manganaro R, Marchetta S, Dulgheru R, llardi F, Sugimoto T, Robinet S, Cimino S, Go YY, Bernard A, Kacharava G, et al. Echocardiographic reference ranges for normal non-invasive myocardial work indices: results from the EACVI NORRE study. Eur Heart J Cardiovasc Imaging. 2019;20(5):582-90.

11. van der Bijl P, Kostyukevich M, El Mahdiui M, Hansen G, Samset E, Ajmone Marsan N, Bax JJ, Delgado V. A roadmap to assess myocardial work: from theory to clinical practice. JACC Cardiovasc Imaging. 2019;12(12):2549-54.

12. Hubert A, Le Rolle V, Leclercq C, Galli E, Samset E, Casset C, Mabo P, Hernandez A, Donal E. Estimation of myocardial work from pressurestrain loops analysis: an experimental evaluation. Eur Heart J Cardiovasc Imaging. 2018;19(12):1372-9.

13. MarwickTH, Gillebert TC, Aurigemma G, Chirinos J, Derumeaux G, Galderisi M, Gottdiener J, Haluska B, Ofili E, Segers P, et al. Recommendations on the use of echocardiography in adult hypertension: a report from the European Association of Cardiovascular Imaging (EACVI) and the American Society of Echocardiography (ASE). J Am Soc Echocardiogr. 2015;28(7):727-54.

14. Pieske B, Tschöpe C, de Boer RA, Fraser A, Anker S, Donal E, Edelmann F, Guazzi MF, Lam M, Lancellotti C, et al. How to diagnose heart failure with preserved ejection fraction: the HFA-PEFF diagnostic algorithm: a consensus recommendation from the Heart Failure Association (HFA) of the European Society of Cardiology (ESC). Eur Heart J. 2019;40(40):3297-317.

15. Solomon SD, Anavekar N, Skali H, McMurray JJ, Swedberg K, Yusuf S, Granger CB, Michelson EL, Wang D, Pocock S, et al. Influence of ejection fraction on cardiovascular outcomes in a broad spectrum of heart failure patients. Circulation. 2005;112(24):3738-44.

16. Gaasch WH, Zile MR. Left ventricular structural remodeling in health and disease: with special emphasis on volume, mass, and geometry. J Am Coll Cardiol. 2011;58(17):1733-40.

17. Schirone L, Forte M, Palmerio S, Yee D, Nocella C, Angelini F, Pagano F, Schiavon S, Bordin A, Carrizzo A. A review of the molecular mechanisms underlying the development and progression of cardiac remodeling. 2017; 2017:3920195.

18. Chen R, Wu X, Shen LJ, Wang B, Ma MM, Yang Y, Zhao BW. Left ventricular myocardial function in hemodialysis and nondialysis uremia patients: a three-dimensional speckle-tracking echocardiography study. PLOS ONE. 2014;9(6):e100265.

19. Hwang HS, Cho JS, Hong YA, Chang YK, Kim SY, Shin SJ, Yoon HE. Vascular calcification and left ventricular hypertrophy in hemodialysis patients: interrelationship and clinical impacts. International journal of medical sciences. 2018;15(6):557-63.

20. Ishimitsu T, Nakano N, Sudo Y, Akashiba A, Takahashi T, Ohta S, Minami J, Matsuoka H. Predictive significance of blood pressure values for the incidence of cardiovascular events in chronic hemodialysis patients. Hypertension research: official journal of the Japanese Society of Hypertension. 2008;31(9):1703-9.

21. Liu C, Yan ZN, Fan L, Huang J, Shen D, Song XT. Layer-specific speckle tracking analysis of left ventricular systolic function and synchrony in maintenance hemodialysis patients. BMC Cardiovasc Disord. 2020;20(1):126.

22. Mentias A, Alashi A, Naji P, Gillinov AM, Rodriguez LL, Mihaljevic T, Suri RM, Grimm RA, Svensson LG, Griffin BP, et al. Exercise capacity in asymptomatic patients with significant primary mitral regurgitation: independent effect of global longitudinal left ventricular strain. Cardiovasc Diagn Therapy. 2018;8(4):460-8.

23. Kim HM, Cho GY, Hwang IC, Choi HM, Park JB, Yoon YE, Kim HK. Myocardial strain in prediction of outcomes after surgery for severe mitral regurgitation. JACC Cardiovasc Imaging. 2018;1 1 (9):1235-44.

24. Karlsen S, Dahlslett T, Grenne B, Sjøli B, Smiseth O, Edvardsen T, Brunvand $H$. Global longitudinal strain is a more reproducible measure of left ventricular function than ejection fraction regardless of echocardiographic training. Cardiovasc Ultrasound. 2019;17(1):18.

25. Voigt JU, Cvijic M. 2- and 3-dimensional myocardial strain in cardiac health and disease. JACC Cardiovasc Imaging. 2019;12(9):1849-63. 
26. Russell K, Eriksen M, Aaberge L, Wilhelmsen N, Skulstad H, Gjesdal O, Edvardsen T, Smiseth OA. Assessment of wasted myocardial work: a novel method to quantify energy loss due to uncoordinated left ventricular contractions. Am J Physiol Heart Circ Physiol. 2013;305(7):H996-1003.

27. London GM. Cardiovascular disease in chronic renal failure: pathophysiologic aspects. Semin Dial. 2003;16(2):85-94.

28. Sun M, Dong Y, Wang Y, Li G, Huang D. Assessment of the left ventricular function in patients with uremia using layer-specific 2-dimensional speckle tracking echocardiography. Medicine. 2019;98(9):e14656.

29. Duchenne J, Turco A, Ünlü S, Pagourelias ED, Vunckx K, Degtiarova G, Bézy S, Cvijic M, Nuyts J, Claus P, et al. Left Ventricular Remodeling Results in Homogenization of Myocardial Work Distribution. Circulation Arrhythmia electrophysiology. 2019;12(5):e007224.

30. Larsen CK, Aalen JM, Stokke C, Fjeld JG, Kongsgaard E, Duchenne J, Degtiarova G, Gheysens O, Voigt JU, Smiseth OA, et al. Regional myocardial work by cardiac magnetic resonance and non-invasive left ventricular pressure: a feasibility study in left bundle branch block. Eur Heart J Cardiovasc Imaging. 2020;21(2):143-53.

31. Galli E, Leclercq C, Hubert A, Bernard A, Smiseth OA, Mabo P, Samset E, Hernandez A, Donal E. Role of myocardial constructive work in the identification of responders to CRT. Eur Heart J Cardiovasc Imaging. 2018;19(9):1010-8.

32. Galli E, Leclercq C, Fournet M, Hubert A, Bernard A, Smiseth OA, Mabo P, Samset E, Hernandez A, Donal E. Value of myocardial work estimation in the prediction of response to cardiac resynchronization therapy. J Am Soc Echocardiogr. 2018;31(2):220-30.

33. Galli E, Vitel E, Schnell F, Le Rolle V, Hubert A, Lederlin M, Donal E. Myocardial constructive work is impaired in hypertrophic cardiomyopathy and predicts left ventricular fibrosis. J Cardiovasc Imaging Invent. 2019;36(1):74-82.

34. Kim SA, Kim MN, Shim WJ, Park SM. Layer-specific dyssynchrony and its relationship to the change of left ventricular function in hypertensive patients. Heart Vessels. 2016;31(4):528-34.

35. Merchant A, Wald R, Goldstein MB, Yuen D, Kirpalani A, Dacouris N, Ray JG, Kiaii M, Leipsic J, Kotha V, et al. Relationship between different blood pressure measurements and left ventricular mass by cardiac magnetic resonance imaging in end-stage renal disease. J Am Soc Hyperten JASH. 2015;9(4):275-84.

36. Kuznetsova T, D'Hooge J, Kloch-Badelek M, Sakiewicz W, Thijs L, Staessen JA. Impact of hypertension on ventricular-arterial coupling and regional myocardial work at rest and during isometric exercise. J Am Soc Echocardiogr. 2012;25(8):882-90.
37. Oberhoffer FS, Abdul-Khaliq H, Jung AM, Zemlin M, Rohrer TR, Abd EI Rahman M. Assessment of left ventricular myocardial work in Turner syndrome patients: insights from the novel non-invasive pressure-strain loop analysis method. Quant Imaging Med Surg. 2020;10(1):15-25.

38. Cameli M, Mondillo S, Solari M, Righini FM, Andrei V, Contaldi C, De Marco E, Di Mauro M, Esposito R, Gallina S, et al. Echocardiographic assessment of left ventricular systolic function: from ejection fraction to torsion. Heart Fail Rev. 2016;21(1):77-94.

39. El Mahdiui M, van der Bijl P, Abou R, Ajmone Marsan N, Delgado V, Bax JJ. Global left ventricular myocardial work efficiency in healthy individuals and patients with cardiovascular disease. J Am Soc Echocardiogr. 2019;32(9):1120-7.

40. Edwards NFA, Scalia GM, Shiino K, Sabapathy S, Anderson B, Chamberlain R, Khandheria BK, Chan J. Global myocardial work is superior to global longitudinal strain to predict significant coronary artery disease in patients with normal left ventricular function and wall motion. J Am Soc Echocardiogr. 2019;32(8):947-57.

41. Choi JO, Cho SW, Song YB, Cho SJ, Song BG, Lee SC, Park SW. Longitudinal $2 \mathrm{D}$ strain at rest predicts the presence of left main and three vessel coronary artery disease in patients without regional wall motion abnormality. Eur J Echocardiog. 2009;10(5):695-701.

42. Hothi DK, Rees L, McIntyre CW, Marek J. Hemodialysis-induced acute myocardial dyssynchronous impairment in children. Nephron Clinical practice. 2013;123(1-2):83-92.

43. Burton JO, Jefferies HJ, Selby NM, McIntyre CW. Hemodialysis-induced cardiac injury: determinants and associated outcomes. Clin J Am Soc Nephrol CJASN. 2009:4(5):914-20.

44. Bouali Y, Donal E, Gallard A, Laurin C, Hubert A, Bidaut A, Leclerca C, Galli E. Prognostic usefulness of myocardial work in patients with heart failure and reduced ejection fraction treated by sacubitril/valsartan. Am J Cardiol. 2020:125(12):1856-62.

45. Valentim Gonçalves A, Galrinho A, Pereira-da-Silva T, Branco L, Rio P, Timóteo AT, Abreu J, Soares RM, Feliciano J, Moreira RI, et al. Myocardial work improvement after sacubitril-valsartan therapy: a new echocardiographic parameter for a new treatment. J Cardiovasc Med (Hagerstown Md). 2020;21(3):223-30.

\section{Publisher's note}

Springer Nature remains neutral with regard to jurisdictional claims in published maps and institutional affiliations.
Ready to submit your research? Choose BMC and benefit from:

- fast, convenient online submission

- thorough peer review by experienced researchers in your field

- rapid publication on acceptance

- support for research data, including large and complex data types

- gold Open Access which fosters wider collaboration and increased citations

- maximum visibility for your research: over $100 \mathrm{M}$ website views per year

At BMC, research is always in progress.

Learn more biomedcentral.com/submissions 